\title{
IC 5063: AGN driven outflow of warm and cold gas ^
}

\author{
R. Morganti ${ }^{1,2}$, J. Holt $^{3}$, L. Saripalli ${ }^{4,5}$, T. A. Oosterloo ${ }^{1,2}$, and C. N. Tadhunter ${ }^{3}$ \\ 1 Netherlands Foundation for Research in Astronomy, Postbus 2, 7990 AA Dwingeloo, The Netherlands \\ e-mail: morganti@astron.nl \\ 2 Kapteyn Astronomical Institute, University of Groningen, Landleven 12, 9747 AD Groningen, The Netherlands \\ 3 Department of Physics and Astronomy, University of Sheffield, Sheffield, S7 3RH, UK \\ 4 Raman Research Institute, CV Raman Avenue, Sadashivanagar, Bangalore 560080, India \\ ${ }^{5}$ CSIRO, Australia Telescope National Facility, PO Box 76, Epping NSW 1710, Australia
}

Received 15 May 2007 / Accepted 13 September 2007

ABSTRACT

\begin{abstract}
We present new ATCA 17- and 24-GHz radio images and ESO-NTT optical spectra of the radio-loud Seyfert galaxy IC 5063, the first galaxy in which a fast $\left(\sim 600 \mathrm{~km} \mathrm{~s}^{-1}\right)$ outflow of neutral hydrogen was discovered. The new radio data confirm the triple radio structure with a central, unresolved flat-spectrum core and two resolved radio lobes with steep spectral index. This implies that the previously detected fast outflow of neutral gas is occurring off-nucleus, near a radio lobe about $0.5 \mathrm{kpc}$ from the core. The ionised gas shows highly complex kinematics in the region co-spatial with the radio emission. Broad and blueshifted $\left(\sim 500 \mathrm{~km} \mathrm{~s}^{-1}\right) \mathrm{emission}$ is observed in the region of the radio lobe, at the same location as the blueshifted H I absorption. The velocity of the ionised outflow is similar to the one found in HI. The first order correspondence between the radio and optical properties suggests that the outflow is driven by the interaction between the radio jet and the ISM. However, despite the high outflow velocities, no evidence is found for the ionisation of the gas being due to fast shocks in the region of the outflow, indicating that photoionisation from the AGN is likely to be the dominant ionisation mechanism.

The outflow rate of the warm (ionised) gas is small compared to that of the cold gas, similar to what is found in other radio galaxies. The mass outflow rate associated with the $\mathrm{H}$ I is in the same range as for "mild" starburst-driven superwinds in ULIRGs. However, in IC 5063, the AGN-driven outflow appears to be limited to the inner kpc region of the galaxy. The kinetic power associated with the $\mathrm{H}$ I outflow is a small fraction $\left(\mathrm{a}\right.$ few $\times 10^{-4}$ ) of the Eddington luminosity of the galaxy but is a significant fraction $(\sim 0.1)$ of the nuclear bolometric luminosity. In IC 5063, the observed outflows may have sufficient kinetic power to have a significant impact on the evolution of the ISM in the host galaxy.
\end{abstract}

Key words. galaxies: Seyfert - galaxies: active - ISM: jets and outflows

\section{Introduction}

Huge amounts of energy are produced through the accretion of material onto the super-massive black hole situated in the centre of an Active Galactic Nucleus (AGN). This energy is released into the surrounding medium in a number of different ways, ranging from collimated radio-plasma jets to UV and X-ray emission. The regions around an AGN are, therefore, highly complex and host a wealth of physical processes. Gas in different phases (atomic, molecular and ionised) is observed in this very hostile environment. This gas, in particular through its kinematics and ionisation, can carry the signatures of the effect of the AGN on its surrounding medium.

The energy released from the nucleus can produce gas outflows with high velocities (thousands of $\mathrm{km} \mathrm{s}^{-1}$ ). This has been observed in many AGN, from Seyfert galaxies to quasars. Gas outflows are detected as blueshifted absorption or emission line wings in optical, UV and X-ray spectra, (see e.g. Crenshaw et al. 2003; Krongold et al. 2003; Elvis et al. 2002; Holt et al. 2006, and references therein). More recently, fast and massive outflows of neutral hydrogen, detected as 21-cm H I absorption against the central regions of radio-loud galaxies, have also been discovered (Morganti et al. 2005a).

^ Based on observations with the ESO-NTT and with the Australia Telescope Compact Array.
All these different types of gaseous outflows are of great interest for a number of reasons. Understanding the driving mechanism(s) of the outflows is crucial for understanding the physical mechanisms at work in the central regions of galaxies with an AGN. Furthermore, massive AGN-driven outflows are now suggested to dramatically affect the evolution of galaxies due to the large amounts of energy they feed back into the interstellar medium (see e.g. Silk \& Rees 1998; di Matteo et al. 2005).

Gaseous outflows can be driven by super-winds associated with large starbursts (Heckman 2002; Rupke \& Veilleux 2005; Rupke et al. 2005; Veilleux et al. 2005). However, in the case of galaxies with an AGN, radiation or wind pressure from the regions near the active super-massive black hole (i.e. a quasar wind) are the likely drivers of the gas outflows detected at X-ray and UV wavelengths. In radio-loud objects, the interaction of the radio plasma with the (rich) gaseous medium in the direct vicinity of the active nucleus can provide another mechanism that can drive the outflows (see also discussion in e.g. Batcheldor et al. 2007).

For the fast outflows of neutral hydrogen many unresolved issues remain, in particular regarding their origin and their quantitative effect on the ISM. To answer these questions, it is crucial to know where the outflows are occurring with respect to the AGN, and to know the characteristics of the gas in other phases (e.g. ionised). These H I outflows occur, in at least some cases, 

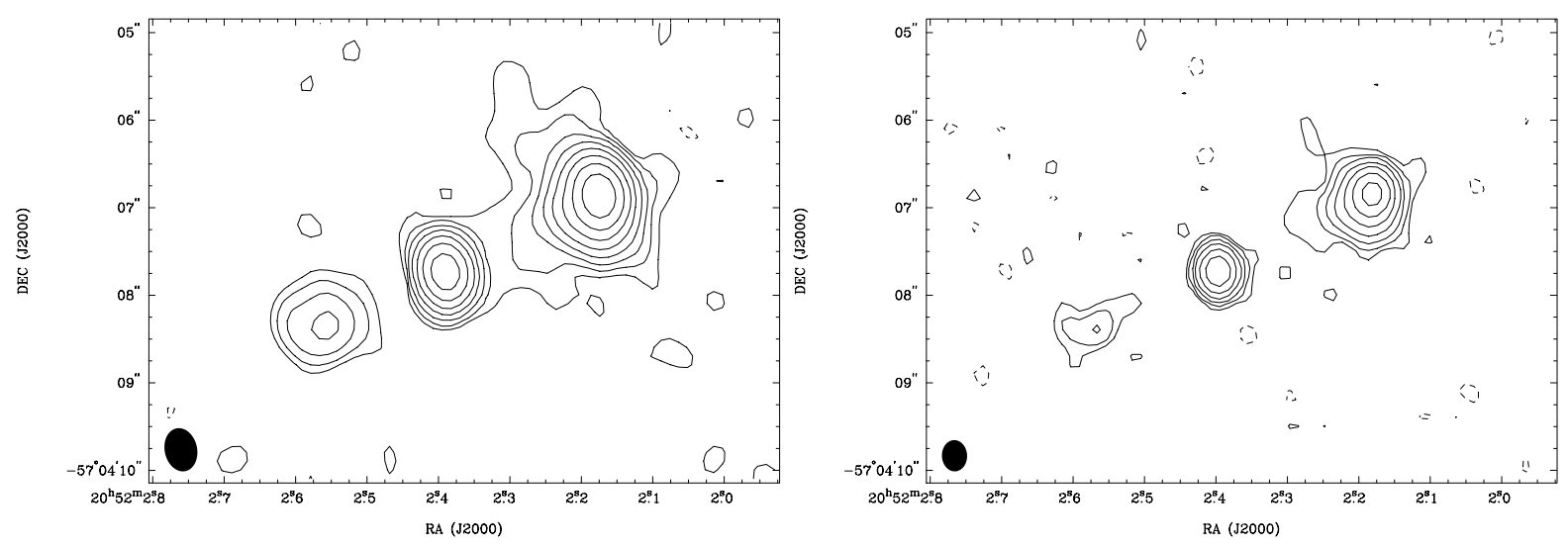

Fig. 1. The radio continuum ATCA images of IC 5063 at $17.8 \mathrm{GHz}$ and $24.8 \mathrm{GHz}$. The contour levels are 0.28 and $0.46 \times-1,1,2,4,8,16$, $32,64,128 \mathrm{mJy} \mathrm{beam}^{-1}$ respectively.

at kpc distance from the nucleus (see Morganti et al. 2005a). This large distance from the AGN suggests that the interaction between the expanding radio jets and the gaseous medium enshrouding the central regions is the driving mechanism of such outflows. The associated mass outflow rates can be as large as $\sim 50 M_{\odot} \mathrm{yr}^{-1}$, comparable (albeit at the lower end of the distribution) to the outflow rates found for starburst-driven superwinds in Ultra Luminous IR Galaxies (ULIRG). This would suggest that these jet-driven outflows of neutral gas in radio-loud AGN can indeed have a significant impact - similar to the one of superwinds - on the evolution of the host galaxies. It is also important to be able to compare the characteristics of the H I outflows with those of the ionised gas. For example, in the radio galaxies 3C 305 and 3C 293 (Morganti et al. 2005b; Emonts et al. 2005; Morganti et al. 2003) the mass of the gas in the ionised outflow is much less than that in the neutral outflow, making the neutral hydrogen the dominant and therefore the most influencing component in the feedback process.

All these considerations motivated a new study of the radioloud Seyfert galaxy IC 5063. IC $5063(z=0.0110)$ is classified as a Seyfert 2 and is classified as an early-type galaxy that shows a complicated system of dust-lanes (see Fig. 1). In polarized light IC 5063 shows high polarization in the near IR (Hough et al. 1987) and a strong, broad $\mathrm{H} \alpha$ emission (Inglis et al. 1993). Like in some other Seyfert 2 galaxies this suggest that there is a broad-line region which is obscured from our direct view and the broad-line radiation is scattered into our line of sight by scatterers outside the obscuring regions. IC 5063 is among the most radio-loud Seyfert galaxies known, while its radio power is at the lower end of the distribution for radio galaxies. IC 5063 is the first galaxy where a fast neutral outflow was discovered (Morganti et al. 1998). In the radio continuum, IC 5063 shows a triple structure of about 4 arcsec in size (about $1.3 \mathrm{kpc}^{1}$; see Morganti et al. 1998) aligned with the dust-lane. The neutral outflow occurs against the NW and strongest radio component (Oosterloo et al. 2000; see also below). With new high-resolution and high-frequency radio observations obtained with the Australia Telescope Compact Array (ATCA) and deep long-slit optical spectra obtained with the ESO New-Technology Telescope (NTT), we aim to study the optical kinematics and ionisation properties in relation to the radio structure. The kinematics (and the ionisation level) of the gas shed light on whether a strong jet/cloud interaction is responsible for the outflow or

${ }^{1}$ We assume $H_{0}=71 \mathrm{~km} \mathrm{~s}^{-1} \mathrm{Mpc}^{-1}$ which implies a distance of $46.5 \mathrm{Mpc} ; 1$ arcsec is equivalent to $0.22 \mathrm{kpc}$. whether other processes must be invoked. This object also allows a detailed comparison between the properties of the ionised-and neutral-gas outflows.

\section{The radio structure: ATCA observations}

IC 5063 was observed with the ATCA using the 6-km configuration - the longest available for this radio telescope. The observations were carried out on 11 May and 8 July 2004 at 17.8 and $24.8 \mathrm{GHz}$ respectively. These high frequencies allow us to achieve a relatively high resolution. We took data simultaneously at 17.73 and $17.86 \mathrm{GHz}$ in the first observation and at 24.77 and $24.90 \mathrm{GHz}$ in the second, with a bandwidth of $128 \mathrm{MHz}$ for each of these frequencies. These separate frequencies allowed us to improve (radially) the $u v$ coverage. For both observations the total integration time on IC 5063 is about $9 \mathrm{~h}$ consisting of many cuts of 10 min spread over a 12-h period. PKS 1934-638 was used as the primary (flux) calibrator (with an assumed flux of $1.079 \mathrm{Jy}$ at $17.8 \mathrm{GHz}$ and $0.745 \mathrm{Jy}$ at $24.8 \mathrm{GHz}$ ). A nearby secondary calibrator (PKS 2052-474) was observed for 1 min every $10 \mathrm{~min}$ as well as for reference pointing. This reference pointing was done every hour.

The data reduction was done using the MIRIAD package (Sault et al. 1995). The final images were obtained using a few cycles of self-calibration and using uniform weighting to achieve the highest possible resolution. The $17-\mathrm{GHz}$ image has a beam size of $0.5 \times 0.37^{\prime \prime}\left(12^{\circ}\right)$ and an rms noise of $0.11 \mathrm{mJy} \mathrm{beam}^{-1}$. At $24 \mathrm{GHz}$ the beam is $0.36 \times 0.29^{\prime \prime}\left(2.5^{\circ}\right)$ with an rms noise of $0.18 \mathrm{mJy}$ beam $^{-1}$.

Figure 2 shows the $17-$ and $24-\mathrm{GHz}$ continuum images. As expected from the previous $8-\mathrm{GHz}$ observations (Morganti et al. 1998), the source shows a triple structure oriented along $\mathrm{PA} \sim 115^{\circ}$. In contrast to previous lower resolution images, the bright NW source appears to be connected to the core by a lowbrightness bridge. Although most of the flux in the NW source appears to come from a quite compact and bright region, lowbrightness, diffuse emission surrounding the NW source is also detected.

The new, higher-resolution observations allow us to investigate in more detail the structure of the radio emission while, due to the broad frequency range of the new observations, we are able to accurately derive the spectral index. The spectral index is crucial information for identifying which of the features correspond to the core and which to radio lobes.

The total fluxes of the three components at the two different frequencies are listed in Table 1. The corresponding spectral 


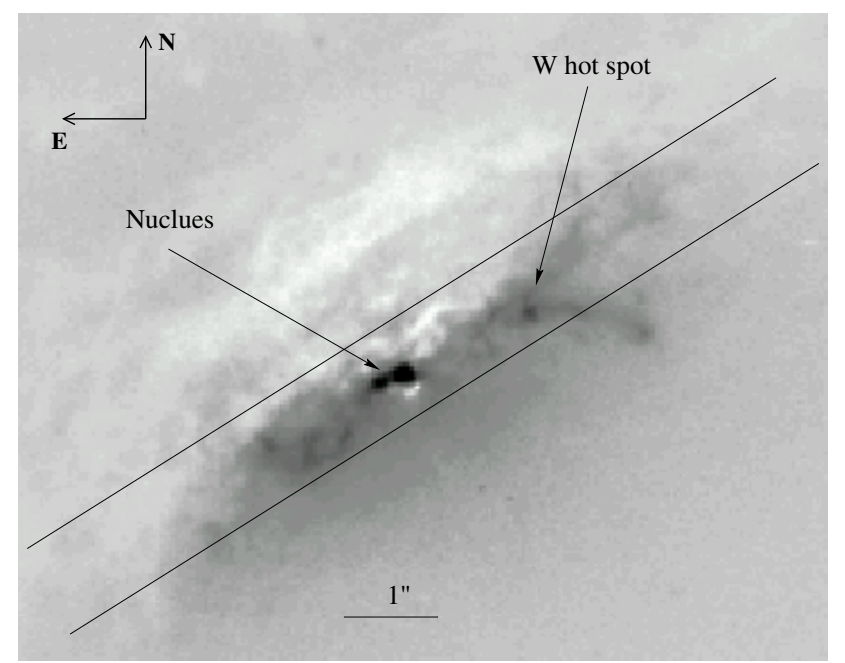

Fig. 2. The slit position of the NTT observations superimposed on the HST image (from the public archive) obtained with WFPC2 through filter F606W (that includes bright emission lines like [O III]).

Table 1. Flux densities and spectral indices for the radio three components in IC 5063.

\begin{tabular}{lccc}
\hline \hline Region & $\begin{array}{c}17 \mathrm{GHz} \\
\mathrm{mJy}\end{array}$ & $\begin{array}{c}24 \mathrm{GHz} \\
\mathrm{mJy}\end{array}$ & $\alpha$ \\
\hline $\mathrm{E}$ & 5.6 & 4.7 & $0.53 \pm 0.02$ \\
core & 30.8 & 29.0 & $0.18 \pm 0.02$ \\
$\mathrm{~W}$ & 91.2 & 67.0 & $0.93 \pm 0.02$ \\
\hline
\end{tabular}

indices ( $\alpha$ defined as $S=v^{-\alpha}$ ) are also listed in this table. In contrast to the $\mathrm{E}$ and $\mathrm{W}$ component, the central component has a flat spectral index $(\alpha=0.2)$, confirming the earlier prediction that this component is the radio core in IC 5063 (Oosterloo et al. 2000). The two east and west extended components show, instead, much steeper spectra, confirming their nature as radio lobes. Thus, the new observations support the interpretation of the radio structure given by Morganti et al. (1998). Given that the neutral hydrogen outflow is detected (via VLBI observations, see Oosterloo et al. 2000) against the stronger (i.e. the western) radio component, the new observations now confirm that this neutral outflow is occurring against a lobe located about $0.5 \mathrm{kpc}$ from the core.

We have derived the polarisation characteristics of the source at the two frequencies. We have obtained images of the Stokes parameters $(Q, U)$, the polarised intensity $\left(P=\left(Q^{2}+U^{2}\right)^{1 / 2}\right)$ and the position-angle of the polarisation $(\chi=0.5 \arctan (U / Q))$. The rms noise of the $Q$ and $U$ images is about 0.08 and $0.1 \mathrm{mJy}^{-1}$ beam $^{-1}$ at 17 and $24 \mathrm{GHz}$ respectively.

The polarised intensity and the fractional polarisation $(m=$ $P / I)$ were estimated only for those pixels for which $P>3 \sigma_{Q U}$. Polarised emission is found mainly over an elongated feature in the western radio lobe, stretching from the total intensity peak to the SW region of the lobe. Fractional polarisation values between $10-20 \%$ are seen towards the SW region at the two frequencies. The fractional polarisation decreases steadily towards the NE with the lowest values of under $1 \%$ measured in the centre of the lobe. Polarised flux is detected only in the western lobe.

In Fig. 3 we have overlayed the position angle distribution of the electric vectors corrected for Faraday rotation on the $17 \mathrm{GHz}$ total intensity map. Using the values of the polarisation position angle at the two frequencies, we derive the rotation measure $(R M)$ over the western lobe. We obtain high $R M$ values

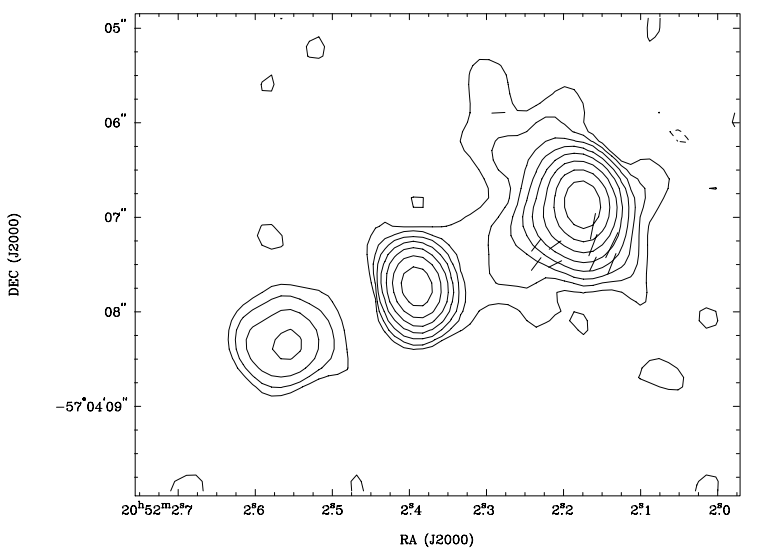

Fig. 3. Vectors proportional to the $17 \mathrm{GHz}$ fractional polarisation (with a $3 \sigma$ cut-off) superimposed on the continuum $17 \mathrm{GHz}$ image. Vector position angle has been corrected for Faraday rotation.

over the region of up to a few thousand $\mathrm{rad} \mathrm{m}^{-2}$ : at $5 \sigma$ level the $R M$ ranges between 2000 and $3000 \mathrm{rad} \mathrm{m}^{-2}$. We do not think the $R M$ derivation suffers from $n \pi$ ambiguities. At these high frequencies, the $R M$ required for a rotation in position angle of $\pi$ radians is about $140000 \mathrm{rad} \mathrm{m}^{-2}-$ a very large and unlikely value.

\section{The ionised gas: NTT observations}

In order to obtain more information about the conditions of the gas in the inner regions of IC 5063, we have investigated the characteristics of the ionised gas using long-slit spectra obtained - in service mode - with the EMMI spectrograph on the 3.5-m New Technology Telescope (NTT) in La Silla, Chile. Using EMMI in medium resolution mode, we obtained simultaneous spectra in the red and in the blue arm. The observations are summarised in Table 2 . In the blue, one central wavelength setting was used $\left(\lambda_{c}=4052 \AA\right)$ to obtain spectra covering 3560-4460 $\AA$. In the red arm, two settings for the central wavelength, $\lambda_{\mathrm{c}}=5150 \AA$ and $\lambda_{\mathrm{c}}=6315 \AA$, were used yielding spectra with useful wavelength ranges of 4450-5760 $\AA$ and 5700-7000 A respectively. Spectra were taken along PA $115^{\circ}$ (see Fig. 2) with a 0.8 -arcsec slit. This PA lies along the major axis of the radio emission as well as the dust-lane and includes both the nucleus and the radio hotspot, $\sim 2 \operatorname{arcsec}$ to the west. The spatial pixel scale is $0.27 \mathrm{arcsec} / \mathrm{pix}$. To reduce the effects of differential refraction, all exposures were taken when IC 5063 was at low airmass $(\sec z<0.1)$.

The data were reduced in the usual way (bias subtraction, flat fielding, cosmic ray removal, wavelength calibration, flux calibration) using the standard packages in IRAF. The twodimensional spectra were also corrected for spatial distortions of the CCD. The final wavelength calibration accuracy, calculated from the locations of the night sky emission lines (Osterbrock et al. 1996) is between 0.05 and $0.1 \AA$ for the red spectra. The spectral resolution estimated from the widths of the night sky emission lines is $2.0-2.4 \AA$ for the red spectra. Due to the absence of sky lines in the blue spectra, we have not been able to quantify the wavelength calibration accuracy nor the spectral resolution in the blue, but there is no reason to believe that it is very different from that in the red spectra.

Comparison of the data of several spectrophotometric standard stars taken with a wide slit ( 5 arcsec) throughout the run gives a relative flux calibration accuracy of about 5 per cent. 
Table 2. Log of observations. Seeing is estimated using the DIMM seeing measurements and service observer's log.

\begin{tabular}{lccccccccc}
\hline \hline $\begin{array}{l}\text { Date } \\
\text { yyyy/mm/dd }\end{array}$ & Arm & $\begin{array}{c}\text { Exposure } \\
\text { s }\end{array}$ & $\begin{array}{c}\text { Set-up } \\
\text { CCD+grating }\end{array}$ & $\begin{array}{c}\text { Slit PA } \\
\text { degrees }\end{array}$ & $\begin{array}{c}\text { Slit width } \\
\operatorname{arcsec}\end{array}$ & $\begin{array}{c}\lambda_{\mathrm{c}} \\
\AA\end{array}$ & $\begin{array}{c}\Delta \lambda \\
\AA\end{array}$ & $\begin{array}{c}\text { Seeing } \dagger \\
\operatorname{arcsec}\end{array}$ & Conditions \\
\hline $2000 / 07 / 25$ & $\mathrm{~B}$ & $4 \times 1800$ & TK1034+\#12 & 115 & 0.8 & 4052 & $3560-4460$ & $0.8-1.0$ & Photometric \\
$2000 / 07 / 25$ & $\mathrm{R}$ & $2 \times 1800$ & TK2048+\#7 & 115 & 0.8 & 5150 & $4450-5760$ & $0.8-1.0$ & Photometric \\
$2000 / 07 / 25$ & $\mathrm{R}$ & $2 \times 1800$ & TK2048+\#7 & 115 & 0.8 & 6351 & $5700-7000$ & $0.8-1.0$ & Photometric \\
\hline
\end{tabular}
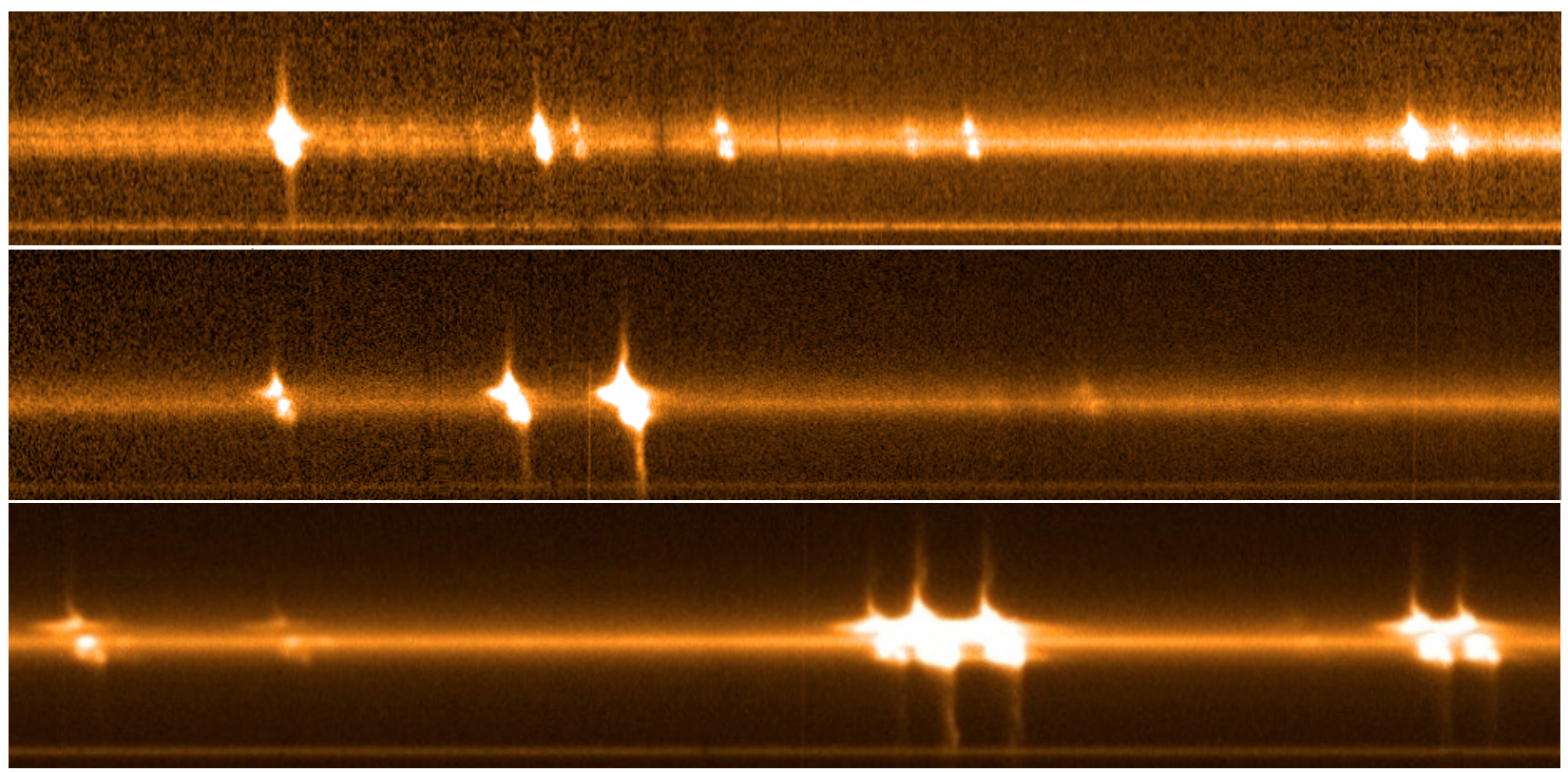

Fig. 4. The spectral regions from [O II] $\lambda \lambda 33727$ to [O III] $\lambda 4363$ (top), H $\beta \lambda 4860$ to [N I] $] 5199$ (middle) and [OI] $\lambda \lambda 6300,6363$ to [S II] $\lambda \lambda 6716,6731$ (bottom) along PA $115^{\circ}$. North-west is to the top, south-east to the bottom. Note, the spectra shown do not have identical scaling in either flux or spatial scale. Rather, the scales are optimised to highlight the differences in the intensity of the lines and allows the different kinematic components to be identified: from regular galaxy rotation at larger radii to the complex kinematics in the nuclear regions, clearly observed in all lines and is highlighted particularly well in the [O I] $\lambda \lambda 6300,6363$ lines in this figure.

This accuracy is confirmed by the good matching of the continuum flux for the different regions.

\subsection{Line fitting and ionised gas kinematics}

Figure 4 shows the 2-dimensional spectra of IC 5063. Due to the very good spatial resolution and the high $\mathrm{S} / \mathrm{N}$, the 2-dimensional spectra were analysed on a pixel-by-pixel, except in the outer regions where the lower $\mathrm{S} / \mathrm{N}$ necessitated binning.

Before proceeding, we would like to stress that in this paper we are mainly going to use the red spectrum and the analysis will focus on the kinematics of the emission lines. The main reason for this is that a proper analysis of the line ratios and diagnostic diagram would require a proper fitting of the underlying stellar continuum (as done in the case of both PKS 1345-12 and PKS 1549-79, Holt et al. 2003, 2006). This is important in order to take into account possible stellar absorption lines (if a young or intermediate stellar population is present, something that cannot be excluded for IC 5063 given the gas rich medium). A first-order discussion of the emission lines ratios is given in Sect. 3.2 while a full analysis is postponed to a future paper.

The spectra were extracted and analysed using the STARLINK packages FIGARO and DIPSO. Following the methods of Holt et al. (2003), we have modelled the emission lines, in each aperture, using multiple Gaussian components. We initially modelled the strong [O III] $\lambda \lambda 4959,5007$ doublet and defined a "good fit" as that comprising the minimum number of Gaussian components to provide a physically viable good fit at each position. We will refer to these models as the "[O III] model" hereafter. The offset velocity is defined relative to the systemic velocity $\left(\sim 3400 \mathrm{~km} \mathrm{~s}^{-1}\right)$ derived from the H I observations (Morganti et al. 1998). Up to four Gaussian components were required in the outflowing region while two or more components were required in other regions. Interestingly, the central region - corresponding to the AGN nucleus - is the least kinematically disturbed region. Examples of the [O III] emission line profiles in different regions are shown in Fig. 5. The location of the radio lobe is also indicated for direct comparison between the characteristics of the ionised gas and the location of the radio emission.

The spatial variations of the velocity widths and shifts and intensity of these components are shown in Fig. 6. We have attempted to group the various components using their kinematical properties to aid in the description below. We realise that the separation in Gaussian components, as well as grouping them into "kinematical components", can be somewhat artificial and subjective. We have based our grouping on kinematical continuity and/or in similarities (e.g. FWHM) of the components. The kinematics of the ionised gas are complex, in particular near the NW radio lobe. We identify the following main kinematic components:

1. As can be seen in Figs. 4 and 5, the emission lines are highly extended along PA $115^{\circ}$. The brightest lines ( $\mathrm{H} \alpha /[\mathrm{N} \mathrm{II}]$, $[\mathrm{O}$ III] $] \lambda \lambda 4959,5007,[\mathrm{O}$ II $] \lambda \lambda 3727$ and $[\mathrm{S} \mathrm{II}] \lambda \lambda 6716 / 6731)$ are observed up to $15.1 \operatorname{arcsec}(3.3 \mathrm{kpc})$ to the $\mathrm{E}$ and 

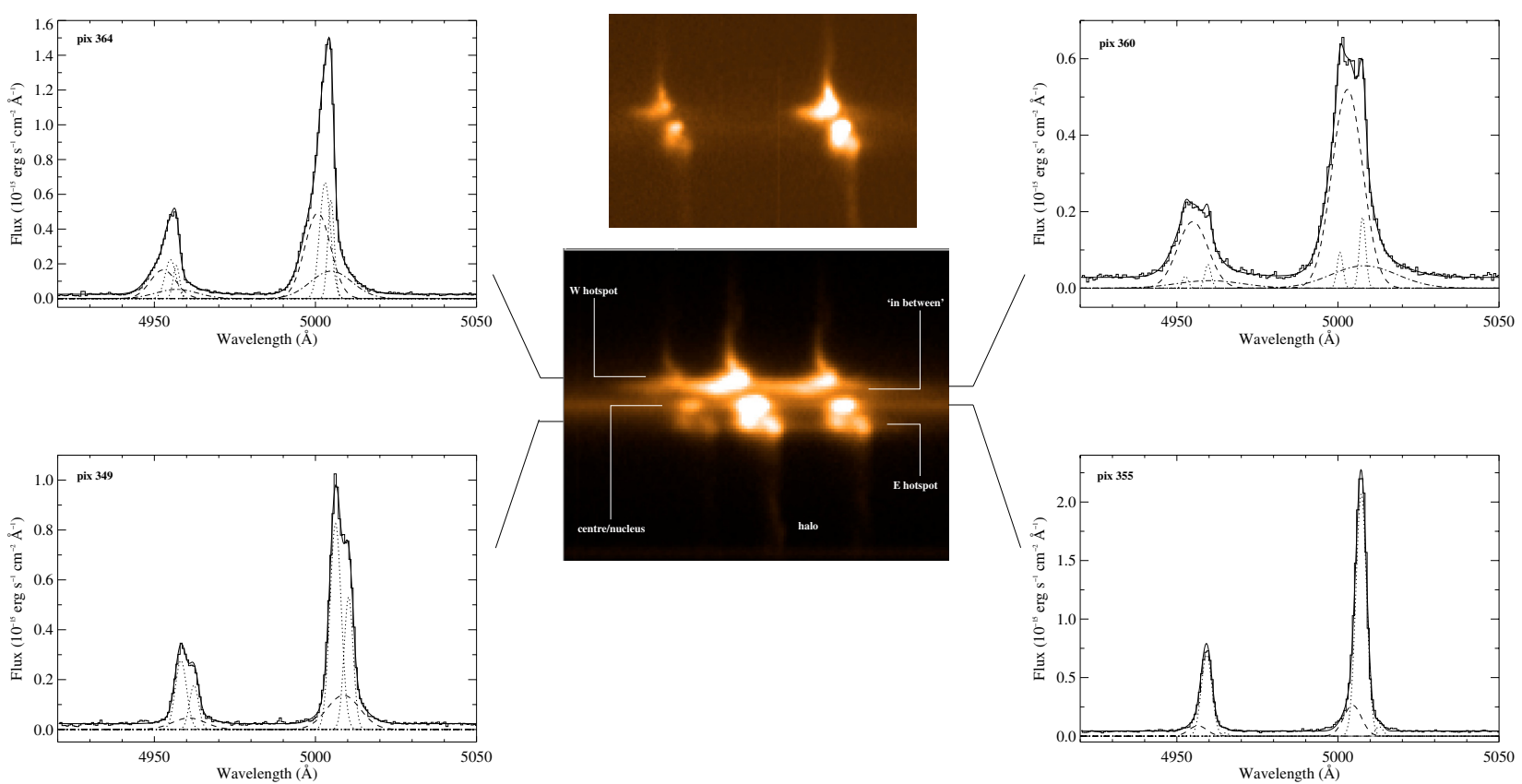

Fig. 5. Spatial variations of the emission line profiles. In the centre is the 2D spectrum, zoomed in on the [O III] $\lambda \lambda 4959,5007$ (top) $\mathrm{H} \alpha / \mathrm{N}$ II blend (bottom). The four regions discussed in Section 4 are identified. On the left and right, a selection of the emission line profiles of [O III] $\lambda \lambda 4959,5007$ at various spatial positions are shown along with the best fitting model and the various sub-components required to model the lines. Key: black line (data), thick line (overall model) dashed lines are the different Gaussian used in the fit, see text for details.
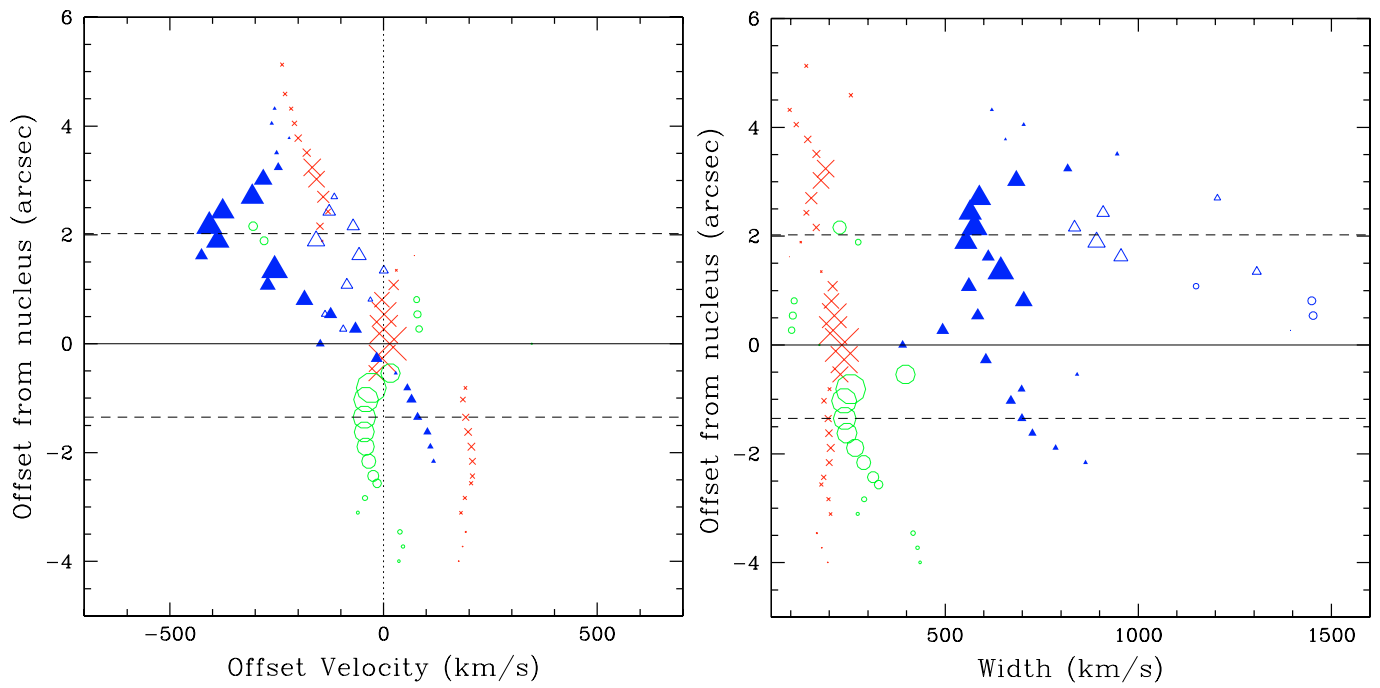

Fig. 6. Spatial variations of the velocity width and shift of the various kinematic sub-components as measured from the [O III] $\lambda \lambda 4959,5007 \mathrm{doublet}$. Crosses (red, in the electronic version) and open circles (green) represent the narrow components, filled triangles represent the extended broad component and open triangles (blue) represent the extremely broad component observed in the western hotspot. In addition, the points are scaled according to the measured line flux in the components. The solid horizontal line marks the position of the nucleus, and the dotted lines mark the position of the western (top) and eastern (bottom) hotspots. The systemic velocity used to estimate the "Offset velocity" is $3400 \mathrm{~km} \mathrm{~s}^{-1}$ as derived from H I observations (Morganti et al. 1998).

$16.2 \operatorname{arcsec}(3.6 \mathrm{kpc})$ to the $\mathrm{W}$ of the core. At these distances from the centre, a narrow component (FWHM 100-200 $\mathrm{km} \mathrm{s}^{-1}$ ), which follows the rotation of the galaxy with velocity amplitude $\sim 200 \mathrm{~km} \mathrm{~s}^{-1}$ is detected (the crosses in Fig. 6).

2. In the region from the nucleus extending to at least 4 arcsec SE and 2 arcsec NW, we detect a second narrow component $\left(F W H M \sim 200-300 \mathrm{~km} \mathrm{~s}^{-1}\right.$, the open circles in Fig. 6) close to the systemic velocity. This component is seen outside the region of radio emission on the $\mathrm{E}$ side.
3. A broad component, $500<F W H M<700 \mathrm{~km} \mathrm{~s}^{-1}$, is observed along the entire spatial extent of the radio emission (the filled triangles in Fig. 6). In the region around the NW lobe (where we see the most extreme kinematics), this broad component appears highly blueshifted $\left(\sim 500 \mathrm{~km} \mathrm{~s}^{-1}\right)$, clearly deviating from the regular kinematics of the galaxy.

4. A very broad component, (up to $\sim 900-1300 \mathrm{~km} \mathrm{~s}^{-1}$ ) only present around the region coincident with the NW radio lobe, and centred on the systemic velocity (i.e. not blueshifted, open triangles in Fig. 6). 
Table 3. Emission lines measured in three main regions. Symbols are as in Fig. 6. The errors listed here are the combination of those associated with the fitting and due to flux calibration error. In bracket are very uncertain values. The fluxes for the SE region are derived from a 0.8 arcsec wide aperture. The NW represents two locations bracketing the hot spot.

\begin{tabular}{|c|c|c|c|c|c|c|c|}
\hline Region & component & $\begin{array}{c}\mathrm{H} \beta \text { flux } \\
10^{-16} \mathrm{erg} \mathrm{s}^{-1} \mathrm{~cm}^{-2} \AA^{-1}\end{array}$ & {$[\mathrm{O} \mathrm{III}] / \mathrm{H} \beta$} & $\mathrm{H} \alpha / \mathrm{H} \beta$ & [S II]6716/6731 & $\begin{array}{c}\text { density } \\
\mathrm{cm}^{-3}\end{array}$ & $E(B-V)$ \\
\hline \multirow[t]{3}{*}{$\mathrm{SE}$} & narrow $(\times)$ & $7.8 \pm 1.2$ & $9.3 \pm 0.2$ & $10.1 \pm 0.2$ & $1.04 \pm 0.05$ & $513 \pm 65$ & 0.94 \\
\hline & narrow (o) & $9.3 \pm 1.2$ & $12.8 \pm 0.1$ & $11.1 \pm 0.1$ & $1.06 \pm 0.05$ & $471 \pm 60$ & 1.02 \\
\hline & broad (4) & $11.6 \pm 3.2$ & $9.4 \pm 0.1$ & $8.1 \pm 0.3$ & $1.1 \pm 0.06$ & $395 \pm 100$ & 0.77 \\
\hline \multirow[t]{3}{*}{ centre } & narrow (o) & $31.0 \pm 2$ & $12.1 \pm 0.1$ & $12.9 \pm 0.1$ & $0.99 \pm 0.05$ & $632 \pm 80$ & 1.14 \\
\hline & narrow $(x)$ & $2.8 \pm 1.5$ & $4.6 \pm 0.5$ & $8.7 \pm 0.5$ & $0.99 \pm 0.19$ & $632_{-365}^{+750}$ & 0.82 \\
\hline & broad (ム) & $(4.1 \pm 2.5)$ & $(31 \pm 1)$ & $(26 \pm 1)$ & $0.94 \pm 0.14$ & $774_{-340}^{+610}$ & 1.70 \\
\hline \multirow[t]{4}{*}{ NW 1} & narrow $(\times)$ & $0.9 \pm 0.6$ & $5.8 \pm 0.6$ & $1.0 \pm 0.6$ & 1.42 & $<100$ & 0.0 \\
\hline & narrow (o) & $1.7 \pm 0.4$ & $8.9 \pm 0.2$ & $4.5 \pm 0.3$ & 1.42 & $<100$ & 0.30 \\
\hline & broad $(\mathbf{\Lambda})$ & $5.0 \pm 0.9$ & $9.7 \pm 0.2$ & $7.5 \pm 0.2$ & $0.5 \pm 0.17$ & $>3000$ & 0.70 \\
\hline & very broad $(\triangle)$ & $5.2 \pm 0.9$ & $6.9 \pm 0.2$ & $8.1 \pm 0.2$ & $1.1 \pm 0.6$ & $395 \pm 52$ & 0.77 \\
\hline \multirow[t]{4}{*}{ NW 2} & narrow $(\times)$ & $2.8 \pm 0.2$ & $6.3 \pm 0.1$ & $3.5 \pm 0.1$ & 1.42 & $<100$ & 0.34 \\
\hline & narrow (o) & $1.2 \pm 0.2$ & $12.8 \pm 0.2$ & $7.6 \pm 0.2$ & 1.42 & $<100$ & 0.72 \\
\hline & $\operatorname{broad}(\mathbf{\Lambda})$ & $4.2 \pm 0.4$ & $13.3 \pm 0.1$ & $9.2 \pm 0.1$ & $0.7 \pm 0.1$ & $2390 \pm 1000$ & 0.87 \\
\hline & very broad $(\Delta)$ & $6.4 \pm 0.4$ & $4.0 \pm 0.1$ & $6.1 \pm 0.1$ & $1.22 \pm 0.06$ & $213 \pm 25$ & 0.54 \\
\hline
\end{tabular}

Hence, it is clear that the kinematics of the ionised gas is very complex. In particular, we note that there is a close correspondence between the ionised gas kinematics and the radio structure while the most extreme ionised gas kinematics occur, to first order, at the location where the fast outflow of neutral gas is detected. All these features suggest that a strong interaction between the radio plasma and the ISM is taking place.

\subsection{Physical conditions of the ionised gas}

A few interesting features can be extracted from the various line ratios observed. We will concentrate on a few relevant regions (see Table 3).

In the nuclear region, many strong emission lines are observed, as previously reported by Colina et al. (1991). We confirm the detection of high-ionisation species such as $[\mathrm{Fe} \mathrm{X}] \lambda 6375$, [Fe VII] $\lambda 5721$ and [Fe VII] $\lambda 6087$. In addition, we observe a feature at $5309 \AA$, similar to that observed in PKS 2152-699 by Tadhunter et al. (1989) which we identify as $[\mathrm{Ca} \mathrm{V}] \lambda 5309$. The region of the SE radio lobe is also characterised by strong, high-ionisation emission lines. In the region of the NW lobe, many bright emission lines are observed, although the highest-ionisation species found in the nucleus and eastern radio hotspot are not observed in this region.

Optical images of IC 5063 show a complex dust lane system (see Fig. 2) and, as our slit was aligned along this dust lane, it is likely that some of the emission line components will be reddened to some degree. We have estimated the degree of reddening in three regions (nucleus, northern outflowing region and the southern region with double narrow lines) using the commonly used Balmer decrement $\mathrm{H} \alpha / \mathrm{H} \beta$. In all regions and in all kinematic components, we measure significant reddening, typically $\mathrm{H} \alpha / \mathrm{H} \beta>6$, corresponding to $E(B-V)>0.5$. In all regions, the largest reddening is found in the component with intermediate blueshift and in one of the two narrow components (see Table 3). Whilst our results are consistent with the presence of a large, patchy dust lane, the specific numbers must be used with caution. No detailed modelling of the optical continuum was done and therefore no corrections for stellar absorption lines were applied to e.g. the $\mathrm{H} \beta$ flux and it is therefore possible that we have underestimated the $\mathrm{H} \beta$ flux due to underlying stellar absorption, and so overestimated the degree of reddening.
We have performed a quick fit to the stellar continuum to estimate the worst case scenario for possible underlying $\mathrm{H} \beta$ absorption. In the southern and northern apertures, the effect of stellar absorption lines is negligible to a few percent and our estimated uncertainties on the emission line fluxes will cover this. In the northern region, the effect is again up to a few percent in the narrow components. However, the effect is perhaps as large as $20-25 \%$ in the intermediate component in the central region. Keeping this uncertainty in mind, we can make a few remarks about the line ratios.

After modelling [O III] $\lambda \lambda 4959,5007$ in the various regions (see Sect. 3.1), we have attempted to model some of the other emission lines (limited to $\mathrm{H} \beta$ and [S II]6716/6731) using the same kinematic model as derived for the [O III] for the same aperture. We fixed the velocity widths and shifts and only allowed the relative fluxes of the kinematic subcomponents to vary.

For one of the narrow components (the circles in Fig. 6), the $[\mathrm{O} \mathrm{III}] / \mathrm{H} \beta$ ratio is high $(\sim 12)$ in the centre. As this component is narrow, it is unlikely to be strongly influenced by the interaction of the radio plasma with the ISM and the ionisation of this gas is likely due to the $U V$ radiation from the nucleus.

High [O III] $/ \mathrm{H} \beta$ ratios (between 10 and 12) are also observed in the narrow components at the location of the radio lobes. Furthermore, high values of the $[\mathrm{O} \mathrm{III}] / \mathrm{H} \beta$ ratio are also observed in the intermediate-width $\left(600 \mathrm{~km} \mathrm{~s}^{-1}\right)$, blueshifted component in the lobes. Even if no detailed analysis of the line ratios is done in this paper for IC 5063, the $[\mathrm{O} \mathrm{III}] / \mathrm{H} \beta$ ratios allow a useful comparison with the detailed studies of jet-cloud interactions in radio galaxies. In the radio galaxies the $[\mathrm{O} \mathrm{III}] / \mathrm{H} \beta$ ratios provide the best evidence for shock ionization: the shocked components tend to have low $[\mathrm{O} \mathrm{III}] / \mathrm{H} \beta$ ratios (see e.g. 3C 171: Clark et al. 1997; PKS 2250-41: Villar-Martin et al. 1999), in contrast to the situation of the kinematically disturbed components to to the NW in IC 5063. Thus, this suggests that even if these components would originate from gas shocked by the interaction between the radio plasma and the ISM, these shocks are not the dominant ionisation mechanism. Although this result will need to be confirmed using more emission lines (and after a proper continuum subtraction), it nevertheless supports the conclusions based on the energy budget (Morganti et al. 1998) and is also 


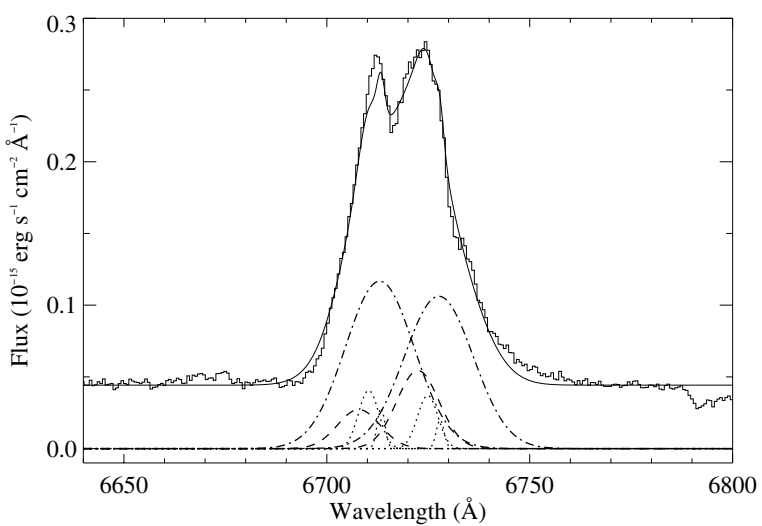

Fig. 7. Example of fitting of the [S II] lines for the broad, blueshifted component $2 \operatorname{arcsec} \mathrm{NW}$ of the nucleus superimposed onto the data. The black line represents the overall model, the dotted line the narrow component, the dashed line the intermediate and the dot-dashed the broad component.

consistent with what has been found in similar studies of other Seyfert galaxies, e.g. Mrk 78 (Whittle et al. 2005).

As with all other strong emission lines in IC 5063, the density diagnostic, the [S II] $\lambda \lambda 6716,6731$ emission line doublet, is spatially resolved enabling us to investigate the density distribution of the emission-line gas. By modelling the doublet with the [O III] model, we derived the density sensitive ratio 6716/6731 in the three regions mentioned earlier (Table 3). The narrow component seems to show a density of at most few hundred particles per cubic centimeter. For the NW region of the fast outflow, the best fit was obtained by setting the weak narrow components to the low density limit. The intermediate-width $\left(\sim 600 \mathrm{~km} \mathrm{~s}^{-1}\right)$, blueshifted component observed in the NW region (as well as the intermediate component in the SE lobe) shows a much larger density $\left(>2000 \mathrm{~cm}^{-3}\right)$. This is consistent with what is expected if these broad line are produced in the region of interaction between the radio plasma and the surrounding medium.

\section{Discussion}

The observations presented in this paper give strong evidence for the idea that the radio plasma ejected by the Seyfert nucleus in IC 5063 is strongly interacting with the ISM of this galaxy. The new, high-frequency radio observations demonstrate that the brightest radio component is indeed a radio lobe. This implies that the fast, outflow of neutral gas is occurring off-nucleus, about $0.5 \mathrm{kpc}$ from the core. The deep long-slit optical spectra show that the kinematics of the ionised gas is very complex over the entire region in which the radio emission is observed. The most extreme kinematics, in the form of a fast outflow of ionised gas, is detected at exactly the same location as the neutral outflow detected in H I $21 \mathrm{~cm}$. On the other hand, despite the complex kinematics, the gas is most likely photoionised by the Seyfert nucleus. We now will discuss various aspects of these observations is some more detail.

\subsection{Radio continuum and HI outflow}

Our new radio observations confirm the triple structure of the Seyfert galaxy IC 5063. From the estimated spectral index, it is clear that the central component corresponds to the radio core, while the other two components are radio lobes with a steep spectral index. The bright NW radio lobe is connected to the core by a faint bridge of radio emission that perhaps is a radio jet. The broad blueshifted H I absorption found in previous H I observations (Morganti et al. 1998) is located - as determined by VLBI observations - against this NW radio lobe (Oosterloo et al. 2000). Our new radio data therefore confirm that the outflow is occurring off-nucleus, i.e. against a radio lobe about $0.5 \mathrm{kpc}$ from the nucleus. In this respect, IC 5063 is similar to other cases studied like 3C 305 and 3C 293 (Morganti et al. 2005b, 2003; Emonts et al. 2005).

The location of the fast outflow suggests that it is more likely caused by the interaction between the radio jet and the ISM than by other mechanisms such as e.g. radiation pressure. The interaction scenario assumes that a particularly rich medium is present around the NW radio lobe. That this is indeed the case is suggested by the detection, using NICMOS, of an asymmetric $\mathrm{H}_{2}$ emission, much stronger near the western lobe (Kulkarni et al. 1998) suggesting that an excess of molecular gas is present on the western side. Such emission is usually interpreted as evidence for fast shocks (for example, if the radio jet has struck a molecular cloud). The large rotation measures found in the W lobe also suggests that a particularly rich medium is present at the location of the western lobe. High values of the $R M$ as found in IC 5063, are also measured in Compact Steep Spectrum sources. Twenty percent of the B3-VLA CSS sample of Fanti et al. (2004) have $R M \gtrsim 1000 \mathrm{rad} / \mathrm{m}^{2}$. In these cases, the Faraday screen most likely responsible for the $R M$ is the magnetised interstellar medium. In most of these cases, the screen must be confined to the nuclear environment. An exception to this seems to be the high-redshift radio galaxy PKS B0529-549 (Broderick et al. 2007) where $R M\left(-9600 \mathrm{rad} / \mathrm{m}^{2}\right)$ has been found. In this object, a gaseous halo is likely responsible for the extreme $R M$. The case of IC 5063 appears to be more similar to that of CSS sources. The presence of the high $R M$ seems to further support the idea that the NW radio lobe is actually hitting a region of rich ISM.

Using the column density of the neutral hydrogen $(1 \times$ $10^{22} \mathrm{~cm}^{-2}$ assuming $T_{\text {spin }}=1000 \mathrm{~K}$ ) derived in Oosterloo et al. (2000), we can estimate the mass of the H I involved in the outflow. Assuming that the absorption covers the NW radio lobe $\left(\sim 1\right.$ arcsec in size), we derive an H I mass of $3.6 \times 10^{6} M_{\odot}$. From the H I data the mass outflow rate can also be derived (see Morganti et al. 2005a). Following Heckman (2002) and Rupke et al. (2002), the mass outflow rate for IC 5063 is estimated to be $35 M_{\odot} \mathrm{yr}^{-1}$. As discussed in that paper, this is a substantial outflow rate that is comparable (albeit at the lower end of the distribution) to that seen for starburst super-winds (Rupke et al. 2002)

\subsection{The ionised gas and the warm outflow}

The optical spectra of IC 5063 confirm the high ionisation and the very complex kinematics of the ionised gas (Colina et al. 1991; Wagner et al. 1989). The region where the complex gas kinematics is observed is co-spatial with the radio emission. This is very similar to what was previously found for other Seyfert galaxies and suggests an effect of the radio plasma on the surrounding medium, either via a direct interaction or via an expanding cocoon that surrounds the radio jet (e.g. Mrk 3, Capetti et al. 1999).

Interesting in the context of gas outflows, is the presence of the highly blueshifted emission lines in the region of the bright NW lobe. We interpret this as a fast gas outflow occurring in this region. Although we do not have a similarly detailed image of the complete region for the H I absorption, we know from the 

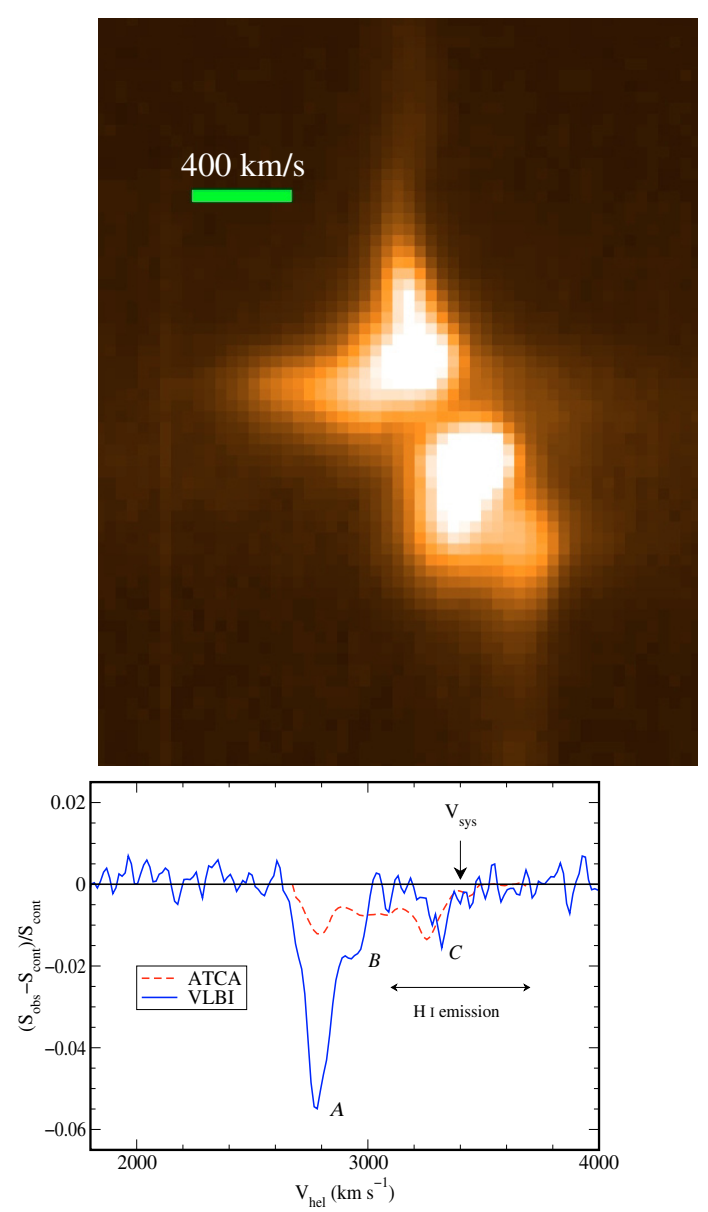

Fig. 8. Comparison between the width of the $\mathrm{H} \mathrm{I}$ absorption (white profile) and that of the ionised gas (from the [O III]5007 $\AA$ ). The first order similarity between the amplitude of the blueshifted component is clearly seen.

VLBI data that the most blueshifted H I absorption component is also seen against the NW radio lobe. The similarity between the kinematics of this blueshifted wing of ionised gas and the $\mathrm{H}$ I is illustrated in Fig. 8. Thus, a fast outflow is observed - at the same location and with similar velocities - in both the atomic neutral and the ionised gas. The fact that these outflows have such similar characteristics indicates that the detection of outflows of neutral hydrogen is likely not biased toward the strong radio continuum (i.e. one would see the outflow occurring only where there is strong background continuum). Nevertheless, we can not entirely rule out the idea that the neutral outflow extends beyond the radio lobes and is driven by a different mechanism (e.g. a starburst-induced super-wind).

Following Holt et al. (2006), we can estimate the mass outflow rate of the warm, ionised gas and compare this with that of the neutral gas. We have determined the $\mathrm{H} \beta$ flux using a large aperture $(1.5 \operatorname{arcsec})$ centred on the outflowing region to include all flux in the outflowing component. This gives a flux in the outflowing component of $\mathrm{H} \beta$ of $(2.34 \pm 0.27) \times$ $10^{-15} \mathrm{erg} \mathrm{s}^{-1} \mathrm{~cm}^{-2} \AA^{-1}$. For the reddening and density, we have used values consistent with those presented in Table 3 for the outflowing region, namely a reddening of $E(B-V)=0.8$ and a gas density of $n_{\mathrm{e}}=2000 \mathrm{~cm}^{-3}$. This gives a reddening corrected $\mathrm{H} \beta$ flux of $(3.53 \pm 0.41) \times 10^{-14} \mathrm{erg} \mathrm{s}^{-1} \mathrm{~cm}^{-2} \AA^{-1}$ and the luminosity of the outflowing component in $\mathrm{H} \beta$ (computed following the method of Holt et al. 2006) is
$L(\mathrm{H} \beta)=9.77 \times 10^{39} \mathrm{erg} \mathrm{s}^{-1} \mathrm{~cm}^{-2}$. A mass outflow rate of $0.08 M_{\odot} \mathrm{yr}^{-1}$ obtained. This is similar to the lower end of the range calculated for PKS $1549-79\left(0.12-12 M_{\odot} \mathrm{yr}^{-1}\right)$. Thus, regardless the uncertainties in the value of the density and as earlier found in other galaxies, the contribution of the outflow of ionised gas is much lower than that of the H I. As discussed in Morganti et al. (2005), the presence of fast neutral outflows indicates that after a strong jet-cloud interaction the gas can cool very efficiently, as is indeed predicted by recent numerical simulations of jets impacting on gas clouds (Mellema et al. 2002; Fragile et al. 2004; Krause 2007). The relatively low outflow rate for the ionised gas suggests that this cooling is indeed efficient and that most of the outflowing gas is neutral.

It is intriguing to see that, while neutral and ionised gas share, to first order, the same location, only a relatively small part of the gas is ionised. Two possibilities can be considered to explain this:

i) a large fraction of the jet-induced outflow is, for example, shielded from the AGN ionizing continuum. We could imagine a situation in which the jet drills through the relatively dense ISM we know to exist in the galaxy, staying relatively confined until it reaches a lower density part of the dust lane structure where the lobes/jets can expand more freely, causing the outflows we observe. Only a small fraction of the outflow is illuminated and ionized by the AGN continuum through the hole drilled out by the initial expansion of the radio jet through the denser inner ISM. In this case, high resolution images of the ionized and neutral outflows would show that they have different detailed spatial distributions, while still both associated with the radio jets;

ii) it is also possible that, following cooling behind the jetinduced shocks, the cooled cloud fragments are relatively large and dense. In this case, the emission lines could be emitted by the outer skins of the clouds photoionised by the AGN, with the masses/volumes of the skins much smaller than the masses/volumes of the cloud fragments as a whole.

Thus, in order to understand what is the situation we will need to image the exact location of the full H I outflow, something that at the moment is not available.

\subsection{Impact of the outflow}

It is important to examine whether the outflows have a significant impact on the ISM of the host galaxy of IC 5063. Given that the H I outflow appears to be the dominant one, in terms of mass outflow rate, we will perform the calculations using the parameters found for the neutral hydrogen. The kinetic power associated with the neutral outflow is of the order of $7 \times 10^{42} \mathrm{erg} \mathrm{s}^{-1}$. This is derived by including the radial and the turbulent component of the flow, the latter estimated from the width of the line (see Holt et al. 2006, for more details). To investigate whether the characteristics of the observed outflows are interesting in the context of the feedback model (Fabian 1999; di Matteo et al. 2005), we can use the values derived from Nicastro et al. (2003) for the black-hole mass and accretion rate of IC 5063. With a black-hole mass of $2.8 \times 10^{8} M_{\odot}$, the Eddington luminosity of IC 5063 is $3.8 \times 10^{46} \mathrm{erg} \mathrm{s}^{-1}$, this means that the kinetic power of the outflow represents about few $\times 10^{-4}$ of the available accretion power. This result is similar to that found for PKS 1549-79 (Holt et al. 2006). However, unlike PKS 1549-79, which accretes at a high Eddington rate, Nicastro et al. (2003) found that IC 5063 accretes at a low rate $(\dot{m} \sim 0.02$, defined as the ratio between 
the nuclear bolometric luminosity and the Eddington luminosity). Thus, in IC 5063 the kinetic power of the outflow appears to be a relative high fraction $(\sim 8 \%)$ of the nuclear bolometric luminosity. Thus, the ratio between radiative luminosity $\left(L_{\mathrm{bol}}\right)$ and the kinetic power in the neutral wind appears to be consistent with that required in the Fabian (1999) model for the quasar feedback effect. However, the overall power in the outflow is much less than envisaged in the numerical simulations of feedback in mergers, in which the quasar is accreting at close to the Eddington rate (e.g. di Matteo et al. 2005). Similar result is also found from the contribution of the warm absorbers as observed in the narrow line Syfert 1 NGC 4051 (Krongol et al. 2007).

\subsection{The SE side}

What is the situation on the other side (i.e. on the SE side) of the nucleus? Also there an intermediate-width component, with velocity width $\sim 600 \mathrm{~km} \mathrm{~s}^{-1}$ is observed co-spatial with the radio emission. This suggests that, although not as extreme as on the NW side, also there the radio plasma interacts with the surrounding medium. As discussed above, the asymmetry seen in the conditions of the medium surrounding the radio source may explain some of the differences both in the radio morphology and in the kinematics of the gas, as these asymmetries are likely to strongly affect the evolution of the radio plasma. We also see a component of ionised gas associated with the regularly rotating large-scale gas disk. In addition to all this, a second component is seen, blueshifted compared to the quiescent gas. It is hard to explain this component only by a jet-cloud interaction and it is perhaps more reminiscent of an expanding cocoon (see e.g. Mrk 3, Capetti et al. 1999). However, in the case of IC 5063, the second component is extending outside the radio emission and this makes the interpretation more complicated. We note that the large-scale gas disk in IC 5063 is seen edge-on, so the line-ofsight is intersecting the disk at various locations, resulting in a large velocity widths and/or line splitting. This might explain the profiles at the SE side.

\section{Conclusions}

We have presented new radio and optical observations of the central regions of the Seyfert galaxy IC 5063. The radio data confirm that the fast outflow of neutral hydrogen detected earlier, is indeed occurring off-nucleus, at about $0.5 \mathrm{kpc}$ from the core. The optical spectra show that the kinematics of the ionised gas deviates strongly from regular rotation in the region coincident with the radio source. This supports the idea that the radio plasma ejected by the Seyfert core is interacting with the ISM of IC 5063. Very broad, blueshifted emissions lines are detected at the location of the NW radio lobe, at the same location at which the neutral outflow is detected, pinpointing the location of the strongest interaction. High rotation measures are detected in the NW lobe, suggesting the presence of a high density medium near the NW lobe.

Despite the strong kinematical evidence for an interaction between the radio plasma and the ISM, we do not find, with the available limited data, evidence for the dominant ionisation being due to this interaction. Instead, photoionisation by the AGN is the most likely ionisation mechanism.

While the mass outflow rate of the neutral gas is substantial and is perhaps large enough for AGN feedback effects to be important in IC 5063, the mass outflow rate in the ionised gas is small compared to that seen in neutral hydrogen. This can be explained by very efficient cooling of the outflowing gas, as proposed in several models for jet-cloud interactions.

Acknowledgements. J.H. acknowledges a PPARC $\mathrm{PhD}$ studentship and a NOVA-Marie Curie Fellowship. She also wishes to thank the Kapteyn Institute, Groningen, for its hospitality where most of this work was done.

\section{References}

Batcheldor, D., Tadhunter, C., Holt, J., et al. 2007, ApJ, in press [arXiv: astro-ph/0702498]

Broderick, J. W., de Breuck, C., Hunstead, R. W., \& Seymour, N. 2007, MNRAS, 375,1059

Capetti, A., Axon, D. J., Macchetto, F. D., Marconi, A., \& Winge, C. 1999, ApJ, 516,187

Clark, N. E., Tadhunter, C. N., Morganti, R., et al. 1997, MNRAS, 286, 558

Colina, L., Sparks, W. B., \& Macchetto, F. 1991, ApJ, 370, 102

Crenshaw, D. M., \& Kraemer, S. B. 2000, ApJ, 532, L101

Crenshaw, D. M., Kraemer, S. B., \& George, I. M. 2003, ARA\&A, 41, 117

Di Matteo, T., Springel, V., \& Hernquist, L. 2005, Nature, 433, 604

Elvis, M., Marengo, M., \& Karovska, M. 2002, ApJ, 576, L106

Emonts, B. H. C., Morganti, R., Tadhunter, C. N., et al. 2005, MNRAS, 362, 931

Fabian, C. 1999, MNRAS, 308, L39

Fanti, C., Branchesi, M., Cotton, W. D., et al. 2004, A\&A, 427, 465

Fragile, P. C., Murray, S., Anninos, P., \& van Breugel, W. 2004, ApJ, 604, 74

Haro-Corzo Sinhué, A. R., Binette, L., Krongold, Y., et al. 2007, ApJ, 662, 145 Heckman, T. M. 2002, in Extragalactic Gas at Low Redshift, ed. J. Mulchaey, \& J. Stocke, ASP Conf. Ser., 254, 292 [arXiv: astro-ph/0107438]

Holt, J. 2005, Ph.D. Thesis, Sheffield University

Holt, J., Tadhunter, C. N., \& Morganti, R. 2003, MNRAS, 342, 227

Holt, J., Tadhunter, C., Morganti, R., et al. 2006, MNRAS, 370, 163

Hough, J. H., Brindle, C., Axon, D. J., Bailey, J., \& Sparks, W. B. 1987, MNRAS, 224,1013

Inglis, M. D., Brindle, C., Hough, J. H., et al. 1993, MNRAS, 263, 895

Krause, M. 2007, New AR, 51, 174

Krongold, Y., Nicastro, F., Brickhouse, N. S., et al. 2003, ApJ, 597, 832

Kulkarni, V., Calzetti, D., Bergeron, L., et al. 1998, ApJ, 492, L121

Mellema, G., Kurk, J. D., \& Röttgering, H. J. A. 2002, A\&A, 395, L13

Morganti, R., Oosterloo, T., \& Tsvetanov, Z. 1998, AJ, 115, 915

Morganti, R., Oosterloo, T., Emonts, B., van der Hulst, J., \& Tadhunter, C. 2003, ApJ, 593, L69

Morganti, R., Tadhunter, C. N., \& Oosterloo, T. 2005a, A\&A, 444, L9

Morganti, R., Oosterloo, T. A., Tadhunter, C. N., van Moorsel, G., \& Emonts, B. 2005b, A\&A, 439, 521

Nelson, C. H., Weistrop, D., Hutchings, J. B., et al. 2000, ApJ, 531, 257

Nicastro, F., Martocchia, A., \& Matt, G. 2003, ApJ, 589, L13

Oosterloo, T., Morganti, R., Tzioumis, A., et al. 2000, AJ, 119, 2085

Osterbrock, D. E., Fulbright, J. P., Martel, A. R., et al. 1996, PASP, 108, 277

Rupke, D. S., \& Veilleux, S. 2005, ApJ, 631, L37

Rupke, D. S., Veilleux, S., \& Sanders, D. B. 2002, ApJ, 570, 588

Rupke, D. S., Veilleux, S., \& Sanders, D. B. 2005, ApJS, 160, 115

Veilleux, S., Cecil, G., \& Bland-Hawthorn, J. 2005, ARA\&A, 43, 769

Villar-Martin, M., Tadhunter, C. N., Morganti, R., Axon, D., \& Koekemoer, A. 1999, MNRAS, 307, 24

Sault, R. J., Teuben, P. J., \& Wright, M. C. H. 1995, in Astronomical Data Analysis Software and Systems IV, ed. R. Shaw, H. E. Payne, \& J. J. E. haynes, ASP Conf. Ser., 77, 433

Silk, J., \& Rees, M. J. 1998, Mon. Not. Astron. Soc., 331, L1

Wagner, S. J., \& Appenzeller, I. 1989, A\&A, 225, L13

Wittle, M., Rosario, D. J., Silverman, J. D., Nelson, C. H., \& Wilson, A. S. 2005, ApJ, 129, 104 